\section{Hemorrhagic bullous colitis in a patient with multiple myeloma}

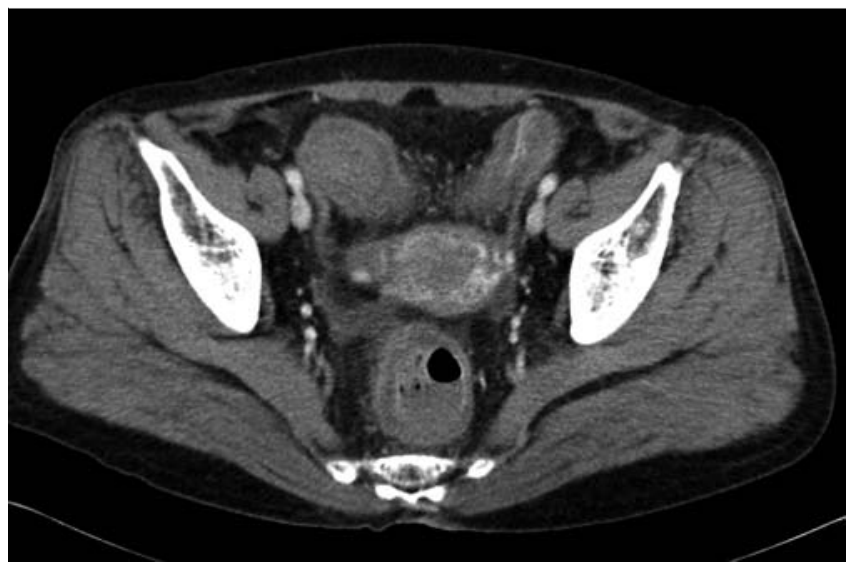

Fig. 1 Contrastenhanced computed tomography (CT) scan in a 65-year-old woman with multiple myeloma showing diffuse edematous wall thickening throughout the entire colon and rectum with some high density lesions visible in the lumen.
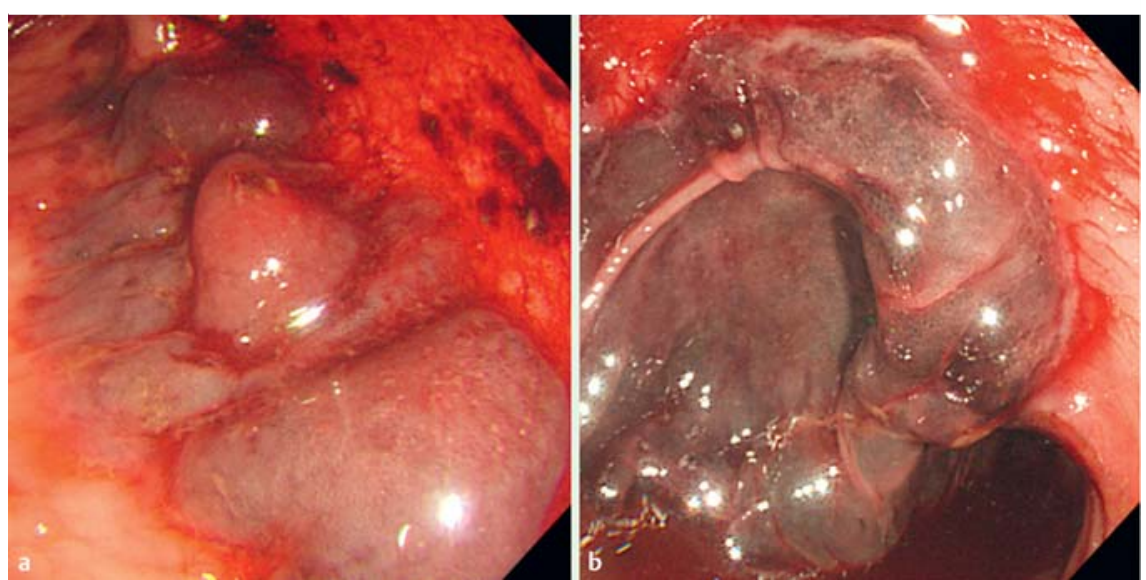

Fig. 2 Endoscopic view of the colon showing multiple bullous hemorrhagic lesions.

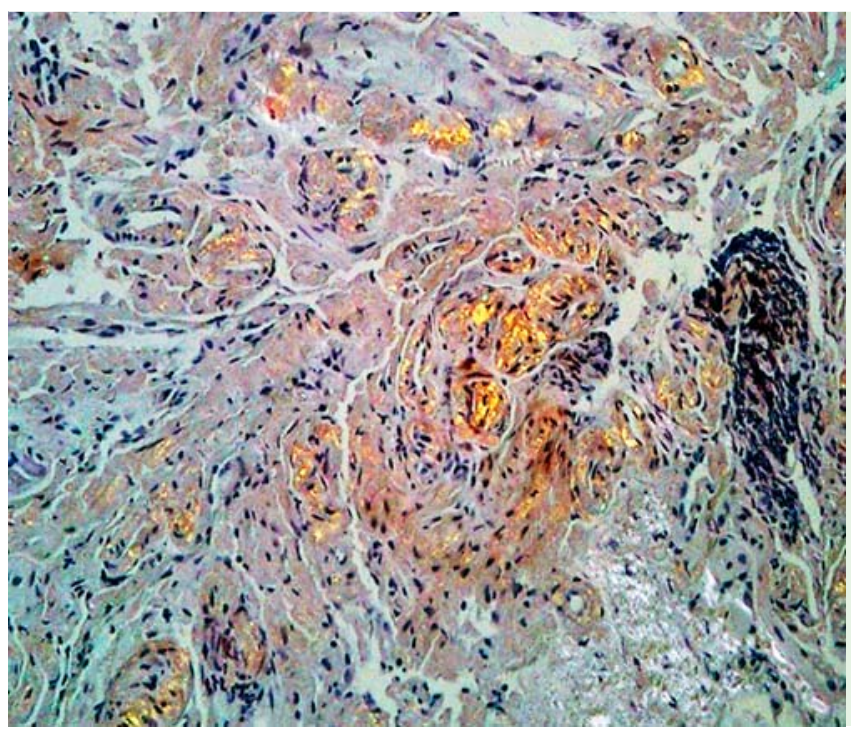

Fig. 3 Histopathologic appearance of a colonic biopsy specimen stained with Congo red and viewed under polarized light showing small foci of birefringence (original magnification $\times 100)$.
A 65-year-old woman diagnosed with multiple myeloma 5 months previously (monoclonal immunoglobulin [Ig]G kappa $26.9 \mathrm{~g} / \mathrm{L}$, dystrophic plasma cells, and bone marrow infiltration) who was being treated with melphalan and prednisolone reported hematochezia and hypogastric pain of 5 hours duration. She had undergone upper and lower gastrointestinal endoscopic examinations 2 years previously with no abnormal findings reported.

She underwent an abdominal computed tomography (CT) scan, which showed diffuse edematous thickening of the colorectal wall with some high density lesions in the lumen, compatible with hemorrhagic colitis ( Fig.1). Rectosigmoidoscopy revealed multiple erosive and bullous hemorrhagic lesions extending from the proximal transverse colon to the rectum ( Fig.2). Colonic biopsies stained with Congo red and viewed under polarized light revealed submucosal birefringent deposits characteristic of amyloidosis ( Fig. 3).

We concluded that the patient was suffering from ischemic colitis secondary to vascular AL amyloidosis that was related to her stage II multiple myeloma. She received treatment with oral tranexamic acid and a monthly intravenous infusion of $4 \mathrm{mg}$ zoledronic acid. Repeat rectosigmoidoscopy 4 months later showed mucosal edema and shallow ulcerations but no evidence of active bleeding or bullous lesions ( $\bullet$ Fig.4).

Amyloidosis is a condition characterized by deposition of an abnormal fibrillar protein between connective tissue cells and parenchymal cells, and within blood vessels [1]. Patients with AL amyloidosis usually present with renal, cardiac, or hepatic involvement and may have underlying multiple myeloma or a lymphoproliferative disorder [2]. Involvement of the colon and rectum are rarely reported and they are thought to be a result of hemorrhage and cleavage between the submucosa and muscularis mucosa due to amyloid deposition and angiopathy [3]. The radiographic manifestations of colonic amyloid are nonspecific and appear to result in part from ischemia.

In a previously reported case, the patient was treated with oral alkylating agents and corticosteroids [4]. This is the first reported case of a patient who was successfully treated with oral tranexamic acid and intravenous zoledronic acid. We have also been able to demonstrate high resolution images of the findings at endoscopy and on CT scanning. 


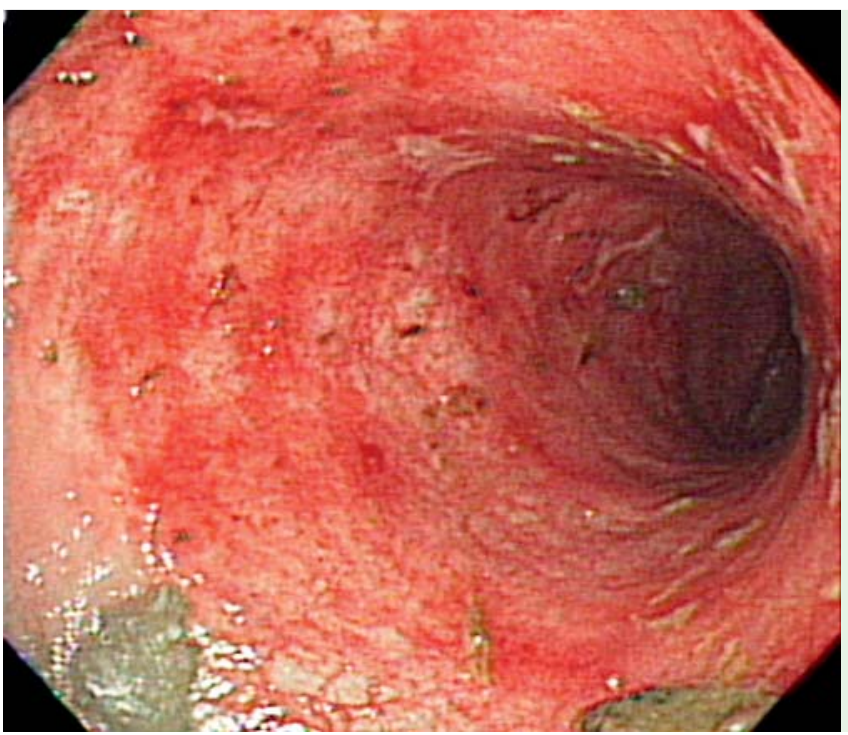

Fig.4 View during rectosigmoidoscopy performed 4 months later showing diffuse mucosal edema and erythematous changes without the bullous hemorrhagic lesions.

Endoscopy_UCTN_Code_CCL_1AD_2AF

\section{Competing interests: None}

\section{S. H. Cho, S. W. Kim, W. C. Kim, L. Chul-Hyun, B. S. Go}

Department of Internal Medicine, The Catholic University of Korea College of Medicine, Seoul, Korea

\section{References}

1 Kyle RA, Gertz MA. Primary systemic amyloidosis: clinical and laboratory features in 474 cases. Semin Hematol 1995; 32: 45 - 59

2 Pérez-Suárez G, Raya JM, Alvarez A et al. Progressive renal failure as the first manifestation of monoclonal light-chain deposition disease with rapid multiple organ involvement. Clin Nephrol 2009; 71: 314-317

3 Yamada M, Hatakeyama S, Tsukagoshi $H$. Gastrointestinal amyloid deposition in AL (primary or myeloma-associated) and AA (secondary) amyloidosis: diagnostic value of gastric biopsy. Hum Pathol 1985; 16 : $1206-1211$

4 Dray X, Treton X, Joly $F$ et al. Hemorrhagic bullous colitis as a primary manifestation of AL amyloidosis. Endoscopy 2006; 38: $15-$ 16

\section{Bibliography}

DOI http://dx.doi.org/

10.1055/s-0032-1326494

Endoscopy 2013; 45: E157-E158

(c) Georg Thieme Verlag KG

Stuttgart · New York

ISSN 0013-726X

\section{Corresponding author}

\section{S. W. Kim, MD}

Division of Gastroenterology, Department of Internal Medicine Seoul St. Mary's Hospital, College of Medicine The Catholic University of Korea 505 Banpo-dong, Seocho-gu Seoul, 137-701

Korea

Fax: +82-2-22582089

alexcho01@hanmail.net 